\title{
Enhanced Sports Image Annotation and Retrieval Based Upon Semantic Analysis of Multimodal Cues
}

\author{
Kraisak Kesorn and Stefan Poslad \\ School of Electronic Engineering and Computer Science, \\ Queen Mary University of London, Mile End Rd, London, E1 4NS, United Kingdom \\ \{kraisak.kesorn, stefan.poslad\}@elec.qmul.ac.uk
}

\begin{abstract}
This paper presents a framework for semi-automatic annotation and semantic image retrieval, applied to the sports domain, based upon semantic analysis of both image text captions and visual features of the image. Unstructured text captions of images are analysed in order to extract the concepts and restructure them into a semantic model. SVM classification of the multi-dominant colours and edge ratio information of the images are used to classify the sport genre. The novelty of the proposed semantic framework is that it can find both the indirectly relevant concepts (concepts not directly referred to) in the visual information and can represent the semantic of images at a higher level by combining image captions and visual feature information. In addition, integrating LSI into the semantic framework enables the proposed system to tolerate ontology imperfections. Experimental results show that the use of the semantic approach significantly enhances image retrieval. Semantic visual information classification and retrieval based upon multimodal cues.
\end{abstract}

Keywords: Ontology, Semantic Model, Image Classification, Knowledge base, Image Retrieval.

\section{Introduction}

Image understanding is one of the most difficult tasks and fastest-growing research areas in the field of computer vision. A huge research effort focuses on the automatic annotation and extraction of visual features which are able to represent semantic of images at the human perception level. The emerging approaches of this task are categorised into two groups [1], data-driven and knowledge-driven. The data-driven approach works on the basis of extracting low-level features and deriving the corresponding high-level content representations without any prior knowledge. While the knowledge-driven approach utilises high-level domain knowledge to extract appropriate content descriptions by guiding features extraction, analysis, and reasoning.

It has been argued that the low-level visual features are not sufficient for depicting the semantic level of the images. This information bears no semantic connection to the actual scene content because it is a simply output of some image processing algorithms. Text and image are two distinct types of information from different modalities [2], as they represent the 'thing' in a quite different way. However, there are some unbreakable and implicit connections between textual and visual information. In the image retrieval 
research area, they can be used to enhance image retrieval by supplementing image content with textual information associated with the image.

In this paper, we propose a novel framework to exploit both low-level features and the associative textual information to perform semantic-based image annotation, indexing, and retrieval. A key feature is that the framework restructures the text captions into the semantic model and tries to predict sport genres using the extracted multi-dominant colours and edge ratio information. These textual and visual cues are combined together in order to narrow the semantic gap and aid the retrieval mechanism. In addition, the hybrid combination of natural language and semantic restructuring degrades nicely when ontology is incomplete as this is compensated by the LSI for natural language processing.

The remainder of this paper is organized as follows: Section 2 analyses state of the art frameworks. Section 3 describes the proposed framework infrastructure. Section 4 describes the implementation and evaluation. Section 5 concludes the strengths, weaknesses, and significance of our approach and, finally, our future work.

\section{Related Work}

There is a vast amount of sport images and videos being produced every day for news, sport, entertainment, and education by media companies and publishers etc. However, sport photograph classification and retrieval using only low-level visual descriptions has proven to be an extremely difficult task to obtain accurate results. Consequently, several techniques were proposed in order to classify and retrieve images at semantic level. Assfalg et al [3] classified sports video by using the playfield colour histogram of the keyframes. However, the keyframes may not contain significant parts of the playfield and the only colour feature is not sufficient to classify different types of sports. Hence, colour, texture, and shape features were deployed to classify sport genres [4]. However, system was limited to distinguishing some sport kinds which have visual similarity e.g., tennis and track events. Multicoloured features of the playing surface and the team uniforms were used to classify sports video in $[5,6]$. In some cases, however, uniform colours are not consistent. Therefore, using uniform colours is unreliable. Edge information was used to classify the sport genres by Yuan [7]. The K-NN algorithm was employed to classify the different sport genres. However, the combination of edge feature with other important visual features is needed in order to achieve more precision and recall.

In fact, even when content-based techniques are applied, textual information surrounding images should not be disregarded since it often includes some form of human generated descriptions of the images which often remain at a higher level to depict the properties that are very difficult to infer by visual features e.g., name of person, time, and place. Visual features, in turn, are useful to classify images into different categories when the associative textual information is not supplied. Therefore, neither textual nor visual information alone can suffice the users' requests. The combination of textual information with image features information has been suggested to improve image search relevance and precision [8], [9], [10]. Wang [11] proposed a data-driven approach for image retrieval using Web images and their textual annotations. By using the data-driven method, the framework is not able to 
search semantically. From the analysis above, we can draw the limitations of the surveyed state-of-the-art frameworks as follows:

\section{1) Image Classification}

1.1) Classification using K-NN technique has some drawbacks. For instance, the computation cost is high because it needs to compute the distance of each query instance to all training samples. In addition, the symmetry problem [12] raises other problems in image classification and automatic annotation.

1.2) Textual information is discarded. Whereas visual information represents content (low-level features) of an image, text captions are useful to describe the context of an image.

1.3) Only green, yellow, and white dominant colours, in some cases, are insufficient to distinguish some sport genres which have similar colour information.

2) Image Retrieval

2.1) The major weakness of the data-driven approach is that it fails to interact meaningfully with users since the built in associations between image semantic and its low-level features quantitative descriptions are not apparent to users. Therefore, the knowledge-driven approach is an alternative approach to solve this problem.

2.2) An image is subjective. A single picture can be interpreted differently by different people. Only visual information cannot support the different views from users.

\section{Proposed Framework}

The framework presented here addresses above limitations and represents the main novelty and contribution of this paper. This section presents a high-level architecture (Fig. 1.) for performing semantics extraction from images based on a predefined semantic model and semantic rules.

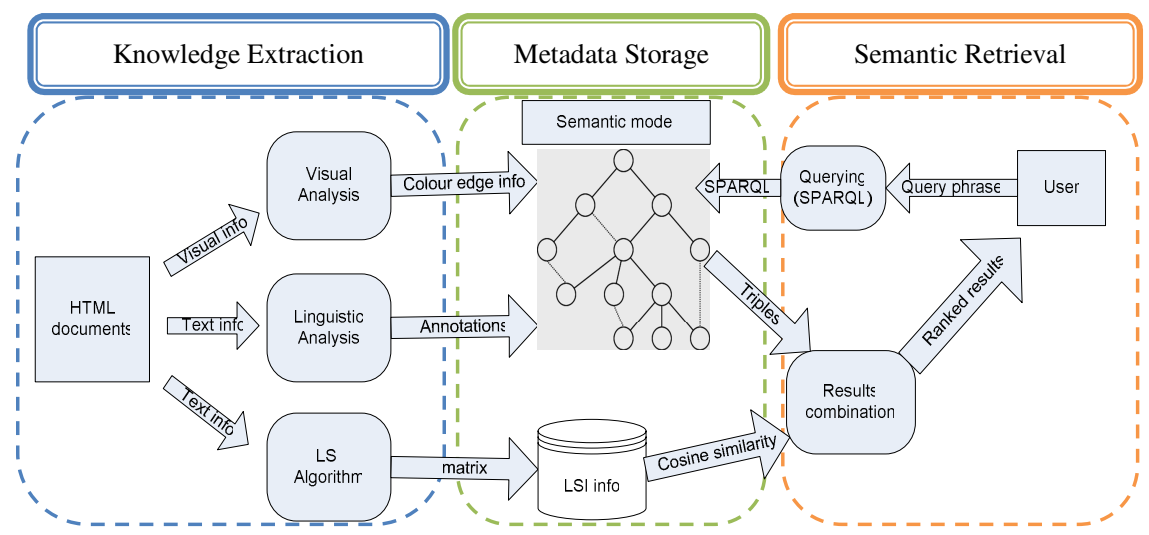

Fig. 1. High-level architecture of the knowledge-based search 


\subsection{Semantic Model}

Among the several knowledge representation formalisms, ontology presents a number of advantages. It provides ways to define well structured concepts and their relationships and subsequently to ease the task of annotation and retrieval. In our framework, two main classes of ontology are defined [13], Domain and Photo annotation ontology. The Domain ontology describes the vocabulary and background knowledge of the photo's subject domain. It comprises two subclasses. The Subject_matter and the Photo_features are created in order to correspond to four main aspects such as what sport type does the photo depict? Who is an athlete in the picture? When and where was the photo made? What is the format of photo? The Photo Annotation Ontology is designed to store the annotations of images in the sport domain. This ontology provides the description template for annotation construction. Fig. 2 depicts the semantic model of the proposed framework.

\subsection{Semantic Linguistic Analysis}

First, the image captions are parsed from HTML documents and, then, a NLP framework, ESpotter [14], processes those text captions. The ESpotter generates an initial version of the annotated documents in the form of XML format. These annotated documents will then be extracted to form the initial metadata and will then be stored in a relational database.

In many cases, an initial metadata entity could match with several ontology entities e.g., the sentence "Kumi Araki from Japan". For computer system, it is difficult to distinguish between 'Japan' which is the 'hostCountry' and 'Japan' which is the 'Nationality' of athletes; therefore, the disambiguation step is required to find the most suitable ontology entity for each metadata. Having been disambiguated, the knowledge discovery step finds any implicit relationship among ontology entities. To do this, semantic rules are applied to this task. Consequently, new metadata may be associated with an image. For instance, if date in the photograph is detected as " 10 February 1998", this picture might have a relationship with the Nagano Olympic Games which took place in year 1998 in Nagano (the host city), and Japan (the host country) and it relates to Winter sport. This solves the data-driven approach limitation in the state of the art frameworks.

The ultimate goal is all semantic metadata will be added to suitable ontology entities. However, some metadata cannot be matched with any particular ontology entities because there is no predefined ontology entity to contain that metadata but it might be important to represent the meaning of an image. Therefore, our system does not discard these non-ontological named entities. They are assigned to the 'otherDetails' ontology entity. On the other hands, some ontology entities might be incomplete by missing the necessary information because that information is not supplied in the text captions. The semantic rules also try to handle the missing information by interlinking the previous relevant semantic metadata and fulfilling any missing ontology information. The following is an example of a simple semantic rule used in our framework. 


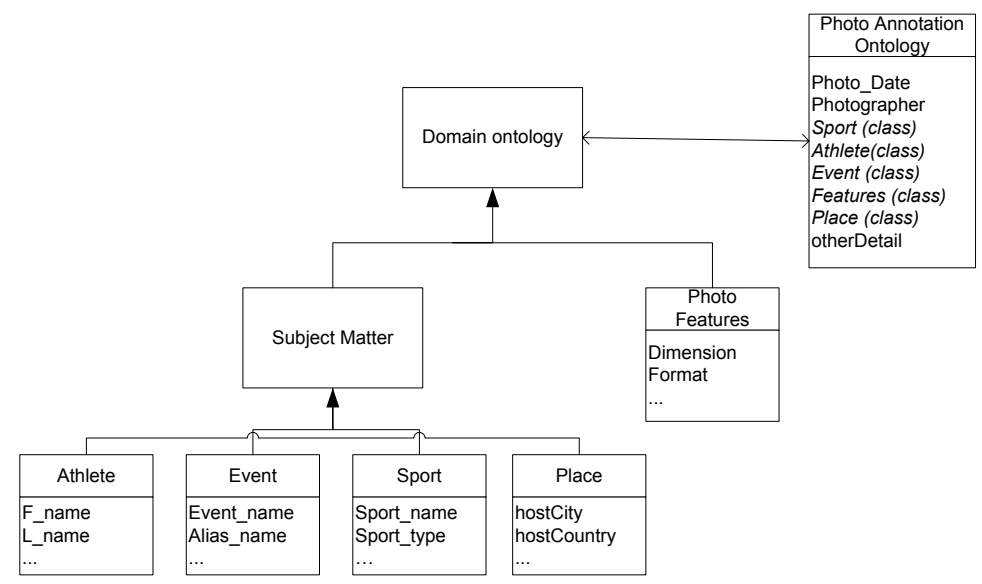

Fig. 2. The semantic model

Add $\mathrm{x}$ to $\mathrm{M}$ (metadata) if all of the following conditions hold:

$$
\begin{aligned}
& \forall x \exists y \mid \operatorname{Photo}(x) \wedge \operatorname{PhotoDate}(x) \wedge \text { happensDuring }(x) \Rightarrow \operatorname{Event}(y) \wedge \\
& \operatorname{hostCity}(y) \wedge \text { hostCountry }(y)
\end{aligned}
$$

Nevertheless, it cannot be expected that the quality of the generated metadata reaches the same quality of manually created metadata. Therefore, manual correction and annotation of metadata are supported. Latent Semantic Indexing (LSI) is also exploited in this framework to solve the ontology imperfection problem. After textual information is parsed from image captions, LSI [15] creates a term-image matrix which contains the numbers of terms (frequency) that appeared with the image. This frequency is used to determine the degree of importance of those terms to the image. Each term will be assigned a weight to show the importance of that term to the image.

\subsection{Semantic Visual Analysis}

Linguistic analysis cannot take place when text captions are not available. To deal with this added uncertainty, the image signature e.g., colour and edge are essential cues to distinguish images. We exploit these features to classify images into different categories of sport types using the Support Vector Machine (SVM) approach.

The HSV (Hue, Saturation, and Value) colour model is intimately related to the way human eye perceives colour. Unlike the previous works, we utilise multidominant colours to classify images rather than using a single dominant colour of playfield $[5,6,7]$. In addition, our framework focuses on the close-up images. It is therefore more challenging to distinguish the sport types in sport images. In order to extract dominant colours, an image will be converted from RGB colour space to HSV colour space using Eq.1 [16]. 


$$
H=\cos ^{-1}\left\{\frac{\frac{1}{2}[(R-G)+(R-B)]}{\sqrt{(R-G)^{2}+(R-B)(G-B)}-}\right\}
$$

Usually, the Hue varies from 0 to 1.0 and is divided into six corresponding colours vary from red through yellow, green, cyan, blue, and magenta. After RGB conversion, every image is normalised and only top three dominant colours which have smallest standard deviation (SD) are selected. As some different sport types have the same dominant colours, edge information is utilised to aid the image classification task. Every image acquires its edge information using the "Canny edge detector" approach. Next, every pixel of an image is examined. The 'edge pixel' is the pixel which has value 1 and non-edge pixel is 0. The edge ratio is calculated by Eq. 2 [7].

$$
\text { edge ratio }=\frac{\text { sum of edge pixels }}{\text { sum of all pixels }}
$$

To consider how good the edge information represent images in different categories, two statistical measures, SD and mean, are exploited to consider the edge information. Mean is an indicator of centre and the SD measures variability. In order to consistently represent the images, the SD should be as small as possible whereas the greater the spread of the mean of image categories, the easier to classify them. Table 1 shows the example of the dominant colours and edge ratio with SD.

In order to achieve fully automatic classification, the SVM is applied for supervised classification. The established SVM [17] is deployed for sport genre classification. The radial basis function $(\mathrm{RBF})$ is used as a kernel function for classification as shown in the following formula:

$$
K\left(x_{i}, x_{j}\right)=\exp \left(-\gamma\left\|x_{i}-x_{j}\right\|^{2}\right), \gamma>0
$$

Where $\gamma$ is a kernel parameter, $\mathrm{x}_{\mathrm{i}}$ and $\mathrm{x}_{\mathrm{j}}$ are training vectors. We select the RBF kernel because it can handle the case when the relationship between class labels and attributes is nonlinear. Furthermore, the RBF kernel has less numerical difficulties.

Table 1. Example of dominant colours, mean, and SD value in different sport categories

\begin{tabular}{|l|r|r|r|}
\hline Sport Categories & $\begin{array}{r}\text { Dominant } \\
\text { Colours }\end{array}$ & $\begin{array}{r}\text { Average Edge } \\
\text { Ratio* }\end{array}$ & $\begin{array}{r}\text { SD of } \\
\text { Edge }\end{array}$ \\
\hline Badminton & Y, B, M & 0.071 & $0.6 \%$ \\
\hline Basketball & Y, B, M & 0.093 & $0.5 \%$ \\
\hline Equestrian & G, B, M & 0.097 & $1.87 \%$ \\
\hline Fencing & G, B, M & 0.067 & $2.20 \%$ \\
\hline Football & R, Y, C & 0.071 & $0.09 \%$ \\
\hline Sailing & R, Y, M & 0.107 & $1.80 \%$ \\
\hline
\end{tabular}

where $\mathrm{R}=\mathrm{Red}, \mathrm{Y}=$ Yellow, $\mathrm{G}=$ Green, $\mathrm{C}=\mathrm{Cyan}, \mathrm{B}=\mathrm{Blue}$, and $\mathrm{M}=$ Magenta 
After images are classified, their annotations (sport genre) are stored in a relational database (RDBMS) and will be processed to find the indirect relationship to other classes in the semantic model using the semantic rules. The methodology for mapping from RDBMS to the semantic model is described in the next section.

\subsection{Semantic Metadata Storage}

The initial semantic metadata generated by the Semantic Linguistic Analysis and the Semantic Visual Analysis is stored in the RDBMS (MySQL). Later, this metadata will be restructured to the semantic model from which data is given a well defined meaning. This enables applications to use data in different contexts. To expose relational database in the semantic model (RDF format), the mapping process [18] is shown in Fig. 3.

1) The initial metadata is retrieved using the SQL select command and the record sets returned from the query are grouped by columns.

2) The Jena API [19] is deployed to create ontology instances and their properties. Jena is a Java class library, and is composed mainly of API and SPI (System Programming Interface).

3) The grouped record set metadata are assigned to the ontology instances created in step 2).

\subsection{Semantic Image Retrieval}

To retreieve images, the query keyword from user will be examined and stop words are eliminated. Then, SPARQL querie is performed. The SPARQL [20] query is executed against the knowledge base, which returns a list of instances that satisfy the query. The cosine similarity algorithm [24] is deployed to compute the similarity between query and image. As shown in Fig. 1, the result of LSI will be used instead of the result from the ontology when the knowledge-based search fails in order to support Ontology imperfection. In other words, the performance of the framework will degrade nicely when the domain ontology is incomplete by compensating using the LSI results.

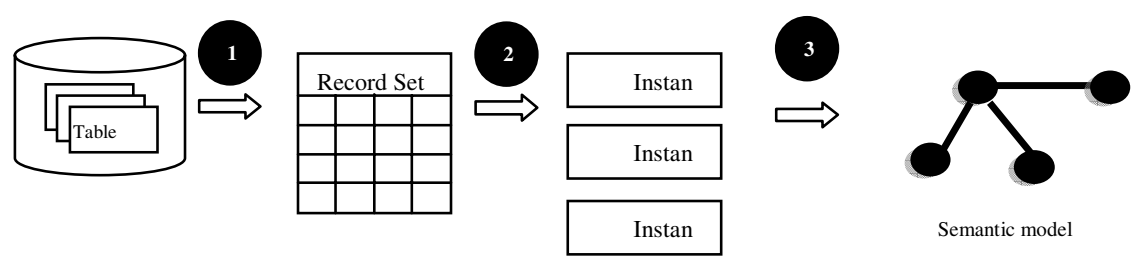

Fig. 3. The RDBMS to RDF mapping

\section{Implementation and Evaluation}

In this experiment, a collection of sport photographs from the Olympic organization website [21] was assembled. To evaluate the retrieval performance, three retrieval 
methods were tested by selected sample queries, and compared the retrieval performance based on the precision and recall values. MTLAB v.7.5 is deployed for visual features extraction and SVM for the classification task.

\subsection{Hypotheses to Evaluate the Retrieval Performance}

Before proceeding to evaluate the classification and image retrieval performance, some hypotheses were established against the limitations of the existing solutions addressed in section 2 .

Hypothesis 1 (H1): the extracted visual information such as dominant colours and edge information are able to distinguish the different types of sport efficiently.

Hypothesis 2 (H2): using the collateral textual information (image captions) and visual information together are able to improve the retrieval performance compared to the text-based and visual-based retrievals.

Hypothesis 3 (H3): the knowledge-driven approach can find implicit relationship among concepts which are not mentioned directly in the text captions but they might be semantically relevant.

Hypothesis 4 (H4): the proposed framework is able to support subjectivity of image by utilising the semantic model.

Hypothesis 5 (H5): the ontology-based search provides good results even though it is imperfect.

Table 2. The confusion matrix of sports classification using SVM approaches

\begin{tabular}{|l|c|c|c|c|c|c|c|c|c|c|c|c|c|}
\hline & Bm & Bb & Bk & Et & Fn & Fb & Sl & Sw & Tn & Rn & Ih & Sk & $\begin{array}{c}\text { Total } \\
\text { images }\end{array}$ \\
\hline Bm & 8 & & & & & & & & 2 & & & & 10 \\
\hline Bb & & 10 & & & & & & & & & & & 10 \\
\hline Bk & & & 10 & & & & & & & & & & 10 \\
\hline Et & & & & 8 & 1 & & & & 1 & & & & 10 \\
\hline Fn & & & & 2 & 8 & & & & & & & & 10 \\
\hline Fb & & & & & & 8 & 1 & & & 1 & & & 10 \\
\hline Sl & & & 2 & & & & 8 & & & & & & 10 \\
\hline Sw & & & & & & & & 10 & & & & & 10 \\
\hline Tn & & & & & & & & & 10 & & & & 10 \\
\hline Rn & & & & & & & 2 & & & 8 & & & 10 \\
\hline Ih & & & & & 1 & & & 1 & & & 8 & & 10 \\
\hline Sk & & & & & & & & & & & & 10 & 10 \\
\hline Accuracy & 8 & 10 & 10 & 8 & 8 & 8 & 8 & 10 & 10 & 8 & 8 & 10 & $106 / 120$ \\
\hline
\end{tabular}

$\mathrm{Bm}=$ Batmiton, $\mathrm{Bb}=$ Baseball, Bk=Basketball, Et=Equestrian, Fn=Fencing, $\mathrm{Fb}=$ Football, Sl=Sailing $\mathrm{Sw}=$ Swimming, Tn=Tennis, Rn=Running, Ih=Ice Hockey, Sk=Skating

\subsection{Classification Performance}

To distinguish the sports genres in images, 120 images were used for training purpose and creating a model by using LIBSVM [17] and another 120 images were used for 
testing. The classification results are shown in Table 2. It is evident that using multidominant colours and the edge ratio information can classify sports efficiently. The overall accuracy the classification model using SVM technique is $88.33 \%$. This statistical information shows that the $\mathrm{H} 1$ hypothesis is clearly verified.

\subsection{Retrieval Performance Measurement}

To evaluate the rest of hypotheses, some sample queries have been selected. Three searching approaches, the keyword-based search (Lucene [22]), the content-based search (LIRe [23]), and the knowledge-based search (the proposed approach), are tested and compared the results. For the content-based search, an image is used as a query instead of keywords. The selected queries are listed as follows:

Query 1 (Q1): Find all photographs of a specific sport type e.g. swimming, football, or basketball. This query is used for the $\mathrm{H} 2$ hypothesis testing. Good image retrieval system should recognise all photographs which both syntactically and semantically related to the query keyword.

Query 2 (Q2): Find all photographs which are semantically relevant to something. For instance, swimming, sailing, and diving are semantically relevant to water. This aims to test the H3 hypothesis. By using the knowledge-driven approach, therefore, the new system should recognize this indirect relevance.

Query 3 (Q3): Find all photographs about specific athlete name. This is a simple query aiming for testing $\mathrm{H} 4$. This is because one might be interested in sport genre in an image whereas other might be interested in an athlete appeared in the picture. Therefore, if same images are returned by using different query keywords, this could imply that the proposed system is able to support the subjective issue.

Query 4 (Q4): Find all photographs of a specific type of sport at specific location e.g., swimming sport in the Sydney (host city). This query aims to test H5 hypothesis. Although a collection does not contain photos about swimming in the Sydney Games, the system should suggest some photographs for the user rather than return nothing to user.

\subsection{Empirical Results and Evaluation}

The framework was tested by 250 photographs. The ontology was created during the knowledge extraction step with more than 2300 instances stored in a RDF file. The experimental results were reported in the 11-point Interpolated Average Precision graphs which are shown in Figure 4 (a) and (b). As Figure $4 \mathrm{a}$ shows, in Q1, the knowledge-based search is superior to the other retrieval approaches. This is because the semantic search supports the expression of more precise information, leading to more accurate answers. Using string matching technique, the keyword-based search has difficulty recognising an image if query keyword is not addressed directly in the image captions. Consequently, it obtains a lower precision and recall. For the content-based search, only visual information cannot represent the meaning of an image precisely. Therefore, it obtains very poor results compared to others. To summarize, using both textual and visual information as the knowledge base there are dramatic improvements in precision and recall. Thus, the $\mathrm{H} 2$ hypothesis could be successfully evaluated. 


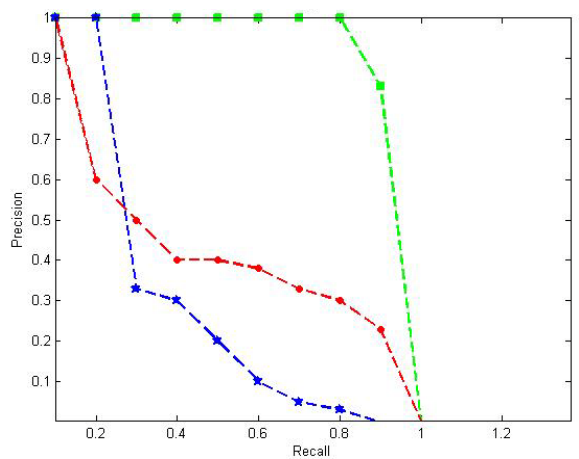

(a)

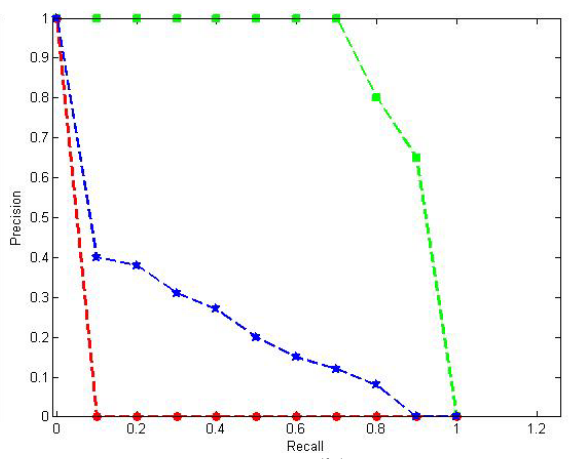

(b)

$$
\begin{aligned}
& \hline-- \text { Knowledge-based search } \\
& -*-\text { Keyword-based search } \\
& -\star-\cdot \text { - Content-based search }
\end{aligned}
$$

Fig. 4. The precision-recall graph for the three retrieval approaches

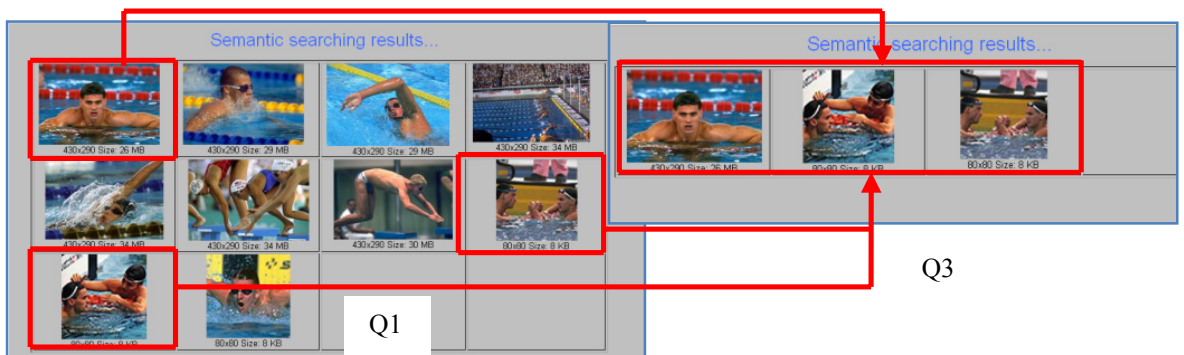

Fig. 5. The result from $\mathrm{Q} 3$ shows the overlapping images with $\mathrm{Q} 1$

To analyse the H3 hypothesis, the Q2 uses a query keyword which does not appear directly in the text captions. As shown in Fig. 4b, the keyword-based search fails because string matching is unsuccessful. The content-based search retrieves all photographs which have similar low-level features which, in many cases, are not semantically relevant to the query image whereas the knowledge-based search performs semantic search by retrieving all photographs which are relevant to a given concept e.g., water. This leads to the knowledge-base search obtaining higher precision and recall. In summary, the knowledge-driven approach improves the retrieval performance significantly and hence confirms the $\mathrm{H} 3$ hypothesis.

The Q3 aims to test the H4 hypothesis. If we analyse the return results shown in Fig. 5, there are some overlapping images between two result sets. Q1 retrieves images for those who are interested in a specific sport genre whereas Q3 searches images in which a particular athlete appeared. It is evident that the proposed system is able to deal with the image subjective issue. Hence, the $\mathrm{H} 4$ hypothesis is clearly validated. Finally, we need a query which fails our search mechanism. I deleted some 


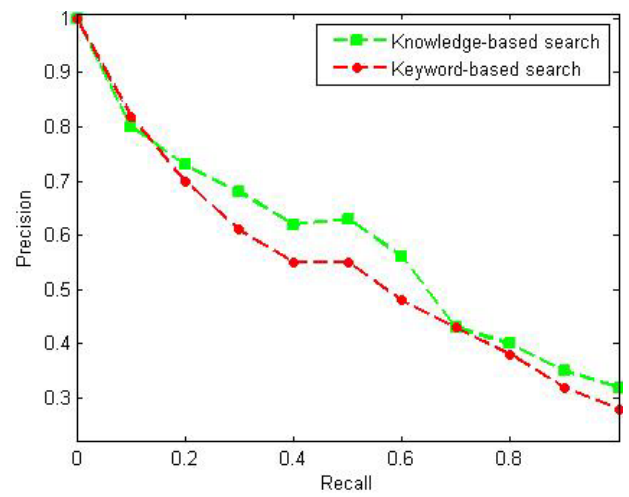

Fig. 6. The result of Q4 comparison for the two approaches

information about swimming in Sydney 2000. We selected the Q4 in order to test H5 hypothesis. Fig. 6 shows that the performance of the knowledge-based search and the keyword-based search are not different. This is because the knowledge-based search alone could not find any photographs which matched to the query. Although the proposed method fails from searching, LSI can find some images which are semantically relevant to the query using its indexing matrix. As a result, the proposed framework obtains higher precision-recall than the keyword search.

\section{Conclusion and Future Work}

This paper has proposed a framework in order to fulfil the limitations of the existing systems analysed in section 2. We utilised the NLP technique to extract and store knowledge in the semantic model. The main innovation is to combine an ontologybased model to restructure the semantic concepts in the natural language captions and visual features. The proposed framework can predict sport genre of an image efficiently when text captions are not supplied. In addition, the knowledge-based search degrades nicely when ontology is incomplete and is compensated by LSI. It is evident from the experimental results that the proposed framework can fulfil the limitations in the state-of-the-art frameworks. We conclude that the main hypotheses of the work, that the (Ontological) knowledge-driven technique can significantly enhance the image retrieval system effectiveness, have been validated.

In the future, several challenges need to be addressed. An appropriate ontology knowledge management technique needs to be investigated so that efficient and effective access and retrieval of the involved knowledge is ensured.

\section{References}

1. Dasiopoulou, S., Spyrou, E., Avrithis, Y., Kompatsiaris, Y., Strintzis, M.G.: Color Image Processing: Methods and Applications. CRC Press / Taylor \& Francis (October 2006)

2. Smeulder, A.W.M., Worring, M., Anntini, S., Gupta, A., Jain, R.: Content-based Image Retrieval at the End of the Early Years. IEEE Trans. Pattern Analysis and Machine Intelligence 22, 1349-1380 (2000) 
3. Assfalg, J., Bertini, M., Colombo, C., Bimbo, A.D.: Semantic Annotation of Sports Video. IEEE Trans. Multimedia 9, 52-60 (2002)

4. Messer, K., Christmas, W., Kittler, J.: Automatic Sports Classification. In: 16th International Conference on Pattern Recognition, vol. 2, pp. 1005-1008 (August 2002)

5. Wang, L., Zeng, B., Lin, S., Xu, G., Shun, H.-Y.: Automatic Extraction of Semantic Colours in Sport Video. In: The International Conference on Acoustics, Speech, and Signal Processing, vol. 3, pp. 617-620 (May 2004)

6. Jang, S., Song, M., Cho, H.: Semantic Classification of Sports News Video Using Colour and Motion Features. In: The 2006 International Conference on Hybrid Information Technology, vol. 2, pp. 745-750 (November 2006)

7. Yuan, Y., Wan, C.: The Application of Edge Feature in Automatic Sport Genre Classification. In: The International Conference on Cybernetic and Intelligent System, vol. 2, pp. 1133-1136 (December 2004)

8. Frankel, C., Swain, M.J., Athitsos, V.: WebSeer: An Image Search Engine for the World Wide Web, Technical Report, The University of Chicago, Illinois (August 1996)

9. Hu, J., Bagga, A.: Categorizing Images in Web documents. IEEE Multimedia 11, 22-30 (2004)

10. Song, X., Ching-Yung, L., Ming-Ting, S.: Autonomous Visual Model Building based on Image Crawling through Internet Search Engines. In: 6th ACM SIGMM international workshop on Multimedia information retrieval (MIR 2004), pp. 315-322 (October 2004)

11. Wang, X., Ma, W., Li, X.: Data-Driven Approach for Bridging the Cognitive Gap in Image Retrieval. In: IEEE International Conference on Multimedia and Expo. (ICME 2004), vol. 3, pp. 2231-2234 (June 2004)

12. Hacid, H., Zighed, A.D.: Semantic-Based Visual Information Retrieval, ch. X. IRM Press, London (2007)

13. Schreiber, A., Dubbeldam, B., Wielemaker, J., Wielinga, B.J.: Ontology-based photo annotation. IEEE Intelligent Systems 16, 66-74 (2001)

14. Zhu, J., ESpotter- Adaptive Named Entity Recognition for Web Browsing, http://kmi.open.ac.uk/people/jianhan/ESpotter

15. Chisholm, E., Kolda, T.G.: New Term Weighting Formulas for The Space Method in Information Retrieval. Computer Science and Mathematics Division (March 1999)

16. Histogram-Based Color Image Retrieval, http://scien.stanford.edu/class/psych221/projects /

17. LIBSVM: A library for support vector machines, http://www.csie.ntu.edu.tw/ cjlin/libsvm

18. D2R Map, http://www4.wiwiss.fu-berlin.de/bizer/d2rmap/ D2Rmap.htm

19. Jena Semantic Web Framework, http://jena.sourceforge.net

20. SPARQL, http://www.w3.org/TR/rdf-sparql-query

21. Olympic organization, http://www. olympic.org

22. Apache Lucene, http: / / lucene. apache.org

23. Lucene Image Retrieval, http: / / www . semanticmetadata.net/wiki/doku.php?id=lire: lire

24. Cosine Similarity and Term Weight Tutorial, http://www.miislita.com/information-retrieval-tutorial/ cosine-similarity-tutorial.html 